\title{
Editorial
}

\section{Disseminated cancer cells in the peritoneal cavity: what can we do when we detect them?}

\author{
YASUHIRO KODERA \\ Department of Surgery II, Nagoya University Graduate School of Medicine, 65 Tsurumai-cho, Showa-ku, Nagoya 466-8550, Japan
}

Cancer recurrence after surgery apparently is due to regrowth of microscopic residual disease that could neither be seen at surgery nor visualized with preoperative imaging studies. Gastric cancer is feared for its capacity to metastasize through various metastatic pathways, of which metastasis through the lymphatic pathway is the most frequently observed. However, it is believed in some countries, including Japan, that a certain degree of lymphatic metastasis can be controlled by conducting extended lymphadenectomy. Most surgeons in Japan have actually seen numerous occasions when gastric cancer with pathologically confirmed nodal metastasis is cured by D2 dissection alone, and are educated to believe that a failure to perform adequate nodal dissection will result in locoregional recurrence. Postoperative irradiation of the gastric bed would therefore be a logical solution to augment a suboptimal surgery, of which D0 dissection is a notable example, and was actually found to significantly improve survival of resectable gastric cancer in North America where locoregional recurrence is commonly observed after the curative surgery [1].

Peritoneal metastasis, on the other hand, is a tough opponent. This could be a consequence of free cancer cells shed from the serosa of the T3 stage cancer. In addition, cancer cells could also be mechanically scraped off from the stomach or may leak out from the lymphatics during surgical manipulation. Peritoneal carcinomatosis has been reported to be the most frequent pattern of disease failure after D2 dissection [2], which, as mentioned above, eradicates the cause for local recurrence. For several decades, Japanese surgeons have been exploring whether detection of free cancer cells in the peritoneal cavity could predict the risk for recurrence and hence lead to any alteration of the treatment strategy for that particular patient. Consequently, the relevance of a histopathologic examination of the peritoneal

Offprint requests to: Y. Kodera washes obtained at surgery was recognized. Results of the examination are currently incorporated in the local stage classification as the CY category, in which CY1 stands for positive cytology and classifies the patient automatically into Stage IV [3]. The prognostic significance of free cancer cells in the peritoneal washes was also observed among patients who were entered onto the Dutch D1 vs D2 trial [4]. However, this was not sufficient to arouse enough interest to cause Western investigators to consider revising the Tumor Node Metastasis (TNM) classification. One of the reasons may have been that accurate diagnosis of peritoneal washing samples through morphology of the floating cells was a formidable task. A more quantitative method may have been preferable but was not available at that time.

Beginning in the early 1990s, several investigators turned to molecular diagnosis by immunostaining and reverse-transcriptase polymerase chain reaction (RTPCR) to detect minimal residual disease and occult metastasis in various body components [5]. Of these, an attempt to apply the RT-PCR technique for detection of free cancer cells in the peritoneal cavity was a success [6] - much more so than detection in the bone marrow aspirates and peripheral blood whose association with clinical outcome was inconsistent for gastric cancer [7]. The relevance of detecting carcinoembyonic antigen (CEA) mRNA in the peritoneal washes as a prognostic determinant and predictor of peritoneal carcinomatosis was confirmed not only through an independent validation study by the same investigators [8] but more soundly among other institutions from numerous countries. The positive predictive value of CEA RT-PCR has not been flawless [6], however, and the pursuit of an optimal target mRNA is ongoing. Several attempts have been reported using various target mRNAs, among which a combination of markers that are less sensitive but highly specific could be the most promising [9]. Ultimately, a good marker or set of markers may in due course be found through gene-profiling studies. Other issues that 
need to be addressed are time and cost required for detection. Use of a LightCycler instrument (Roche Diagnostics, Mannheim, Germany) significantly reduced the time needed to obtain results when compared with conventional thermal cyclers [6], but the transcriptionreverse transcription concerted reaction is reported to be capable of even faster detection [10]. Thus, developments in various aspects of molecular biology allow researchers to constantly renew and improve the methodology. A final product for use in clinical practice remains unavailable, however, and is eagerly awaited.

At long last, a group has been recognized in the United States that has an interest in the issue of free cancer cells in the peritoneal cavity. A report of their attempt, employing the CEA RT-PCR technique similar to what numerous investigators have reported, is published in the current issue of Gastric Cancer [11]. In addition to CEA, they explored three target mRNAs with which they were less successful. Based on their promising results, they now intend to expand their research. Although they were the last to join the crowd, they may be the first to make this examination commercially available, such is the power of their country once they really get on board a project. The American Joint Committee on Cancer (AJCC) seems enthusiastic about incorporating novel prognostic markers in the staging system, and they will have an influence on the International Union against Cancer (UICC) in revising the TNM classification. It is hoped that the RT-PCRmediated detection of intraperitoneal cancer cells may in the future be considered an option to augment conventional diagnostic tools for staging gastric cancer.

There is one small concern in the United States regarding their success. The researchers have explored peritoneal washes collected immediately after laparotomy. As stated previously, cancer cells could also leak out from the lymphatics during surgical manipulation, especially when suboptimal surgery such as D0 dissection is performed. These cells could be detected only by exploring the peritoneal washes collected after gastrectomy. By that time, however, the peritoneal cavity will be contaminated by blood and other body fluids, and specific detection of cancer cells through the RT-PCR technique would become increasingly difficult. When their study expands to include various community hospitals, the American investigations may suffer from a greater incidence of false negative results. Because the authors' institution is reputed for performing D2 dissection, they may need to address this issue by obtaining samples from other hospitals that are less enthusiastic about lymphadenectomy for gastric cancer.

Finally, it may be questioned whether the information obtained through molecular detection should be used solely to identify patients who are not indicated for surgery. Evidence in postoperative adjuvant therapies suggests that isolated cancer cells and micrometastases are more vulnerable to cytotoxic agents than bulky disease. Because the access of anticancer drugs to the peritoneal cavity is limited when given intravenously, lessons could be learned from other types of cancer, and the efficacy of intraperitoneal administration could be explored in the future [12]. Pharmacokinetics of taxanes administered intraperitoneally is well documented, and prolonged exposure to therapeutic concentration of the anticancer agent could eliminate minute quantities of intraperitoneal cancer cells in the absence of visible deposits. It is to be hoped that information obtained through molecular detection may be used eventually to identify candidates who should be treated for cure with some form of multimodal therapy in addition to radical surgery, and not merely to discourage a surgeon from operating on them.

\section{References}

1. Macdonald JS, Smalley SR, Benedetti J, Hundahl SA, Estes NC, Stemmermann GN, et al. Chemoradiotherapy after surgery compared with surgery alone for adenocarcinoma of the stomach or gastroesophageal junction. N Engl J Med 2001;345:725-30.

2. Maehara Y, Hasuda S, Koga T, Tokunaga E, Kakeji Y, Sugimachi K. Postoperative outcome and sites of recurrence in patients following curative resection of gastric cancer. Br J Surg 2000;87:353-7.

3. Japanese Gastric Cancer Association. Japanese classification of gastric carcinoma - 2nd English edition. Gastric Cancer 1998;1: $10-24$.

4. Bonenkamp JJ, Songun I, Hermans J, van de Velde CJ. Prognostic value of positive cytology findings from abdominal washings in patients with gastric cancer. Br J Surg 1996;83:672-4.

5. Wolfrum F, Vogel I, Fandrich F, Kalthoff H. Detection and clinical implications of minimal residual disease in gastro-intestinal cancer. Langenbecks Arch Surg 2005;390:430-41.

6. Kodera Y, Nakanishi H, Ito S, Mochizuki Y, Ohashi N, Yamamura $\mathrm{Y}$, et al. Prognostic significance of intraperitoneal cancer cells in gastric carcinoma: analysis of real time reverse transcriptase-polymerase chain reaction after 5 years of followup. J Am Coll Surg 2006;202:231-6.

7. Kodera Y. Disseminated cancer cells in the bone marrow: are they really there at all? Gastric Cancer 2006;9:243-4.

8. Ito S, Nakanishi H, Kodera Y, Mochizuki Y, Tatematsu M, Yamamura Y. Prospective validation of quantitative CEA mRNA detection in peritoneal washes in gastric carcinoma patients. Br J Cancer 2005;93:986-92.

9. Mori K, Suzuki T, Uozaki H, Nakanishi H, Ueda T, Matsuno Y, et al. Detection of minimal gastric cancer cells in peritoneal washings by focused microarray analysis with multiple markers: clinical implications. Ann Surg Oncol 2007;14:1694-702.

10. Ohashi N, Nakanishi H, Kodera Y, Ito S, Mochizuki Y, Koike M, et al. Intraperitoneal quantitative detection of CEA mRNA in the peritoneal lavage of gastric cancer patients with transcription reverse-transcription concerted (TRC) method. A comparative study with real-time quantitative RT-PCR. Anticancer Res 2007;27:2769-77.

11. Dalal KM, Woo Y, Kelly K, Galanis C, Gonen M, Fong Y, et al. Detection of micrometastases in peritoneal washings of gastric cancer patients by the reverse transcriptase polymerase chain reaction. Gastric Cancer 2008;11:206-13.

12. Armstrong DK, Bundy B, Wenzel L, Huang HQ, Baergen R, Lele $\mathrm{S}$, et al. Intraperitoneal cisplatin and paclitaxel in ovarian cancer. N Engl J Med 2006;354:34-43. 